ISSN 1855-3966 (printed edn.), ISSN 1855-3974 (electronic edn.)

ARS MATHEMATICA CONTEMPORANEA 17 (2019) 67-78

https://doi.org/10.26493/1855-3974.1426.212

(Also available at http://amc-journal.eu)

\title{
On the generalized Oberwolfach problem
}

\author{
Andrea C. Burgess * \\ Department of Mathematics and Statistics, University of New Brunswick, \\ 100 Tucker Park Rd., Saint John, NB E2L 4L5, Canada \\ Peter Danziger ${ }^{\dagger}$ \\ Department of Mathematics, Ryerson University, \\ 350 Victoria St., Toronto, ON M5B 2K3, Canada \\ Tommaso Traetta ${ }^{\ddagger}$ \\ DICATAM, University of Brescia, via Branze 43, 25123 Brescia, Italy
}

Received 19 June 2017, accepted 22 April 2019, published online 20 June 2019

\begin{abstract}
The generalized Oberwolfach problem $\mathrm{OP}_{t}\left(2 w+1 ; N_{1}, N_{2}, \ldots, N_{t} ; \alpha_{1}, \alpha_{2}, \ldots, \alpha_{t}\right)$ asks for a factorization of $K_{2 w+1}$ into $\alpha_{i} C_{N_{i}}$-factors (where a $C_{N_{i}}$-factor of $K_{2 w+1}$ is a spanning subgraph whose components are cycles of length $N_{i} \geq 3$ ) for $i=1,2, \ldots, t$. Necessarily, $N=\operatorname{lcm}\left(N_{1}, N_{2}, \ldots, N_{t}\right)$ is a divisor of $2 w+1$ and $w=\sum_{i=1}^{t} \alpha_{i}$.

For $t=1$ we have the classic Oberwolfach problem. For $t=2$ this is the well-studied Hamilton-Waterloo problem, whereas for $t \geq 3$ very little is known.

In this paper, we show, among other things, that the above necessary conditions are sufficient whenever $2 w+1 \geq(t+1) N, \alpha_{i}>1$ for every $i \in\{1,2, \ldots, t\}$, and $\operatorname{gcd}\left(N_{1}, N_{2}, \ldots, N_{t}\right)>1$. We also provide sufficient conditions for the solvability of the generalized Oberwolfach problem over an arbitrary graph and, in particular, the complete equipartite graph.
\end{abstract}

Keywords: 2-factorizations, resolvable cycle decompositions, cycle systems, (generalized) Oberwolfach problem, Hamilton-Waterloo problem.

Math. Subj. Class.: 05C51, 05C70

\footnotetext{
* The author gratefully acknowledges support from NSERC Discovery Grant RGPIN-435898-2013.

${ }^{\dagger}$ The author gratefully acknowledges support from NSERC Discovery Grant RGPIN-2016-04178.

$\ddagger$ The author gratefully acknowledges the support of a Marie Curie fellowship of the Istituto Nazionale di Alta Matematica, and the support of GNSAGA.

E-mail addresses: andrea.burgess@unb.ca (Andrea C. Burgess), danziger@ryerson.ca (Peter Danziger), tommaso.traetta@unibs.it (Tommaso Traetta)
} 


\section{Introduction}

We denote by $V(G)$ and $E(G)$ the vertex set and the edge set of a simple graph $G$, respectively. Also, we denote by $t G$ the vertex-disjoint union of $t>0$ copies of $G$.

A factor $F$ of $G$ is a spanning subgraph of $G$, namely, a subgraph of $G$ such that $V(F)=V(G)$; also, if $F$ is $i$-regular, we call $F$ an $i$-factor. In particular, a 1-factor of $G$ (also called a perfect matching) is the vertex-disjoint union of edges of $G$ whose vertices partition $V(G)$, while a 2 -factor of $G$ is the vertex-disjoint union of cycles whose vertices span $V(G)$. A 2-factor of $G$ containing only one cycle is usually called a Hamiltonian cycle. We say that a factor is uniform when its components are pairwise isomorphic. Hence, a 1-factor is uniform, whereas a 2-factor might not be.

As usual, we denote by $K_{v}$ the complete graph on $v$ vertices; also, we use $K_{v}^{*}$ to denote the graph $K_{v}$ when $v$ is odd and $K_{v}-I$, where $I$ is a 1 -factor of $K_{v}$, when $v$ is even. Further, we denote by $K_{s}[z]$ the complete equipartite graph with $s$ parts of size $z$. Note that, $K_{v}^{*} \simeq K_{v}[1]$ or $K_{v / 2}$ [2], according to whether $v$ is odd or even, respectively. Finally, we denote by $C_{\ell}$ a cycle of length $\ell \geq 3$ (briefly, an $\ell$-cycle), and by $\left(x_{0}, x_{1} \ldots, x_{\ell-1}\right)$ the $\ell$-cycle with edges $x_{0} x_{1}, x_{1} x_{2}, \ldots, x_{\ell-1} x_{0}$. A uniform 2 -factor whose cycles have all length $\ell$ is referred to as a $C_{\ell}$-factor.

A 2-factorization of a simple graph $G$ is a set $\mathcal{F}$ of 2 -factors of $G$ whose edge sets partition $E(G)$. If $\mathcal{F}$ contains only $C_{\ell}$-factors, we speak of a $C_{\ell}$-factorization of $G$. It is well known that a regular graph has a 2 -factorization if and only if every vertex has even degree. However, if we specify $t 2$-factors, say $F_{1}, F_{2}, \ldots, F_{t}$, and ask for the factorization $\mathcal{F}$ to contain $\alpha_{i}$ factors isomorphic to $F_{i}$, then the problem becomes much harder. Much attention has been given to the cases where $t \in\{1,2\}$ and either $G=K_{v}^{*}$ or $G=K_{s}[z]$.

For $t=1$, we have the "classic" Oberwolfach problem, which is well known to be hard. A survey of the most relevant results on this problem, updated to 2006, can be found in [15, Section VI.12]. For more recent results we refer the reader to [6, 9, 11, 29].

Although the Oberwolfach problem is still open, it has been completely solved for uniform factors when $G=K_{v}^{*}[2,3,22]$ or when $G$ is the complete equipartite graph [24]. We recall these results below.

Theorem 1.1 ([2, 3, 22, 24]). Let $\ell, s$ and $z$ be positive integers with $\ell \geq 3$. There exists $a C_{\ell}$-factorization of $K_{s}[z]$ if and only if $\ell \mid s z,(s-1) z$ is even, further $\ell$ is even when $s=2$, and $(\ell, s, z) \notin\{(3,3,2),(3,6,2),(3,3,6),(6,2,6)\}$.

For $t \geq 1$, we refer to this problem as the generalized Oberwolfach problem. More precisely, given a simple graph $G$, given $t$ 2-factors of $G$, say $F_{1}, F_{2}, \ldots, F_{t}$, and given $t$ non-negative integers $\alpha_{1}, \alpha_{2}, \ldots, \alpha_{t}$, the generalized Oberwolfach problem, denoted by $\mathrm{OP}_{t}\left(G ; F_{1}, F_{2}, \ldots, F_{t} ; \alpha_{1}, \alpha_{2}, \ldots, \alpha_{t}\right)$, or briefly by $\mathrm{OP}_{t}\left(G ;\left(F_{i}\right) ;\left(\alpha_{i}\right)\right)$, asks for a factorization of $G$ into $\alpha_{i} F_{i}$-factors for $i \in\{1,2, \ldots, t\}$. In the case where each $F_{i}$ is uniform, namely, $F_{i}$ is a $C_{N_{i}}$-factor, we denote the problem by $\operatorname{OP}_{t}\left(G ; N_{1}, N_{2}, \ldots, N_{t}\right.$; $\left.\alpha_{1}, \alpha_{2}, \ldots, \alpha_{t}\right)$, or briefly by $\operatorname{OP}_{t}\left(G ;\left(N_{i}\right) ;\left(\alpha_{i}\right)\right)$. Further, we use $v$ in place of $G$ when $G=K_{v}^{*}$. The following necessary conditions are trivial.

Theorem 1.2. If there exists a solution to $\mathrm{OP}_{t}\left(G ;\left(N_{i}\right) ;\left(\alpha_{i}\right)\right)$, then the following conditions hold:

(1) $G$ is regular of degree $2 \cdot \sum_{i=1}^{t} \alpha_{i}$,

(2) $\operatorname{lcm}\left(N_{1}, N_{2}, \ldots, N_{t}\right)$ is a divisor of the order of $G$. 
The case in which $t=2$ is known as the Hamilton-Waterloo problem. Although it has received much interest recently, it is still open even in the uniform case. Some of the most important results up to 2006 can be found in [15, Section VI.12]. More recent results can be found in $[4,7,8,10,12,13,16,23,25]$. For more details and some history on the problem, we refer the reader to $[12,13]$.

Much less is known on $\mathrm{OP}_{t}\left(v ;\left(F_{i}\right) ;\left(\alpha_{i}\right)\right)$ when $t>2$. In [1, 18, 19] the problem is solved for odd orders $v$ up to 17, and even orders $v$ up to 10 (see also [15, Sections VI.12.4 and VII.5.4]). In [6] the problem is settled whenever $v$ is even, each $F_{i}$ is bipartite (namely, $F_{i}$ contains only cycles of even length), $\alpha_{1} \geq 3$ is odd, and the remaining $\alpha_{i}$ are even. In $[14,17]$ the problem is solved whenever $v=p^{n}$ with $p$ a prime number, $t=n$, and $F_{i}$ is a $C_{p^{i}}$-factor, except possibly when $p$ is odd and the first non-zero integer of $\left(\alpha_{1}, \alpha_{2}, \ldots, \alpha_{n}\right)$ is 1 . A partial asymptotic existence result has recently been given in [20], provided that $v$ is sufficiently large and $\alpha_{1}$ scales linearly with $v$. Further results covering specific cases can be found in $[5,26,28]$.

In this paper, we focus on the "uniform" generalized Oberwolfach problem $\mathrm{OP}_{t}(v$; $\left.\left(N_{i}\right) ;\left(\alpha_{i}\right)\right)$. In view of Theorem 1.2, for such a problem to be solvable $v$ must be a multiple of each $N_{i}$ and $\left\lfloor\frac{v-1}{2}\right\rfloor=\sum_{i=1}^{t} \alpha_{i}$; clearly, $1 \leq t \leq \frac{v-1}{2}$. Since $\mathrm{OP}_{t}\left(v ;\left(N_{i}\right) ;\left(\alpha_{i}\right)\right)$ has been solved for $t=1$ (Theorem 1.1), from now on we assume that $t>1$. Also, we denote by $[a, b]$ the set of integers from $a$ to $b$ inclusive; clearly, $[a, b]$ is empty when $a>b$.

The main result of this paper is the following.

Theorem 1.3. Let $v \geq 3$ be odd, let $3 \leq N_{1}<N_{2}<\cdots<N_{t}$ and set $N=$ $\operatorname{lcm}\left(N_{1}, N_{2}, \ldots, N_{t}\right)$ and $g=\operatorname{gcd}\left(N_{1}, N_{2}, \ldots, N_{t}\right)$; also, let $\alpha_{1}, \alpha_{2}, \ldots, \alpha_{t}$ be positive integers. Then, $\mathrm{OP}_{t}\left(v ;\left(N_{i}\right) ;\left(\alpha_{i}\right)\right)$ has a solution if and only if $N$ is a divisor of $v$ and $\sum_{i=1}^{t} \alpha_{i}=\frac{v-1}{2}$ except possibly when $t>1$ and at least one of the following conditions is satisfied:

(I) $\alpha_{i}=1$ for some $i \in[1, t]$;

(II) $\alpha_{i} \in\left[2, \frac{N-3}{2}\right] \cup\left\{\frac{N+1}{2}\right\}$ for every $i \in[1, t]$;

(III) $g=1$;

(IV) $v=N$.

Given a graph $G, G[n]$ denotes the lexicographic product of $G$ with the complement of $K_{n}$, namely, $G[n]$ is the graph whose vertex set is $V(G) \times \mathbb{Z}_{n}$, and two vertices $(x, j)$ and $\left(y, j^{\prime}\right)$ are adjacent if and only if $x$ and $y$ are adjacent in $G$.

The proof of the main theorem relies on the solvability of $\mathrm{OP}_{t}\left(C_{g}[n] ;\left(g n_{i}\right) ;\left(\alpha_{i}\right)\right)$. More precisely, we prove the following result.

Theorem 1.4. Let $t \geq 1$ and let $1 \leq n_{1}<n_{2}<\cdots<n_{t} \leq n$ be odd integers such that $n_{i}$ is a divisor of $n$ for each $i \in[1, t]$. Then $\mathrm{OP}_{t}\left(C_{g}[n] ;\left(g n_{i}\right) ;\left(\alpha_{i}\right)\right)$ has a solution whenever $g \geq 3, \sum_{i=1}^{t} \alpha_{i}=n$, and $\alpha_{i} \geq 2$ for every $i \in[1, t]$.

In the next section we introduce some tools and provide some powerful methods which we use in Section 3 where we prove Theorem 1.4. In Section 4 we prove the main results.

\section{Preliminary results}

We will make use of the notion of a Cayley graph on an additive group $\Gamma$, not necessarily abelian. Given $\Omega \subseteq \Gamma \backslash\{0\}$, the Cayley graph $\operatorname{Cay}(\Gamma, \Omega)$ is a graph with vertex set 
$\Gamma$ and edge set $\{\gamma(\omega+\gamma) \mid \gamma \in \Gamma, \omega \in \Omega\}$. When $\Gamma=\mathbb{Z}_{n}$ this graph is known as a circulant graph. We note that the edges generated by $\omega \in \Omega$ are the same as those generated by $-\omega \in-\Omega$, so that $\operatorname{Cay}(\Gamma, \Omega)=\operatorname{Cay}(\Gamma, \pm \Omega)$, and that the degree of each point is $|\Omega \cup(-\Omega)|$.

Given a subgraph $G$ of $\operatorname{Cay}(\Gamma, \Omega)$ and an element $\gamma \in \Gamma$, we denote by $G+\gamma$ the translate of $G$ by $\gamma$, that is, the graph obtained from $G$ by replacing each of its vertices, say $x$, with $x+\gamma$. It is not difficult to check that $G+\gamma$ is a subgraph of Cay $(\Gamma, \Omega)$. For a subgroup $\Sigma$ of $\Gamma$, the orbit of $G$ under $\Sigma$ (briefly, the $\Sigma$-orbit of $G$ ) is the set $\operatorname{Orb}_{\Sigma}(G)$ of all distinct translates of $G$ by an element of $\Sigma$, that is, $\operatorname{Orb}_{\Sigma}(G)=\{G+\sigma \mid \sigma \in \Sigma\}$. The

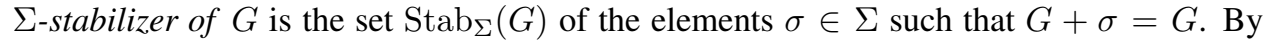
the well-known orbit-stabilizer theorem (see [27, Theorem 5.7]), $\operatorname{Stab}_{\Sigma}(G)$ is a subgroup of $\Sigma$ of index $\operatorname{Orb}_{\Sigma}(G)$, and therefore $\left|\operatorname{Orb}_{\Sigma}(G)\right| \cdot\left|\operatorname{Stab}_{\Sigma}(G)\right|=|\Sigma|$.

Given a set $\Omega \subseteq \Gamma$, we denote by $C_{\ell}[\Omega](\ell \geq 3)$ the graph with point set $\mathbb{Z}_{\ell} \times \Gamma$ and edges $(j, \gamma)(1+j, \omega+\gamma)$, with $j \in \mathbb{Z}_{\ell}, \gamma \in \Gamma$ and $\omega \in \Omega$. In other words, $C_{\ell}[\Omega]=$ $\operatorname{Cay}\left(\mathbb{Z}_{\ell} \times \Gamma,\{1\} \times \Omega\right)$; hence, it is $2|\Omega|$-regular. It is straightforward to see that if $\Gamma$ has order $n$, then $C_{\ell}[n] \cong C_{\ell}[\Gamma]$; hence, $C_{\ell}[\Omega]$ is a subgraph of $C_{\ell}[n]$. We call the elements of $\Omega$ (mixed) differences.

Finally, given a set of cycle factors, $\mathcal{C}$, of $C_{\ell}[n]$, and a set $\Omega \subseteq \Gamma$ we say that $\mathcal{C}$ exactly covers $\Omega$, or $C_{\ell}[\Omega]$, if $\mathcal{C}$ is a factorization of $C_{\ell}[\Omega]$.

The following result, which generalizes Theorem 2.11 of [13], provides sufficient conditions for the existence of a solution to $\mathrm{OP}_{t}\left(C_{\ell}[\Omega] ;\left(\ell n_{i}\right) ;\left(\alpha_{i}\right)\right)$, where $\Omega$ is a subset of an arbitrary group $\Gamma$ of order $n$ and each $n_{i}$ is a positive divisor of $n$.

Theorem 2.1. Let $\Gamma$ be an additive group of order $n$ not necessarily abelian, and let $1 \leq$ $n_{1}<n_{2}<\cdots<n_{t} \leq n$ be odd integers such that $n_{i}$ is a divisor of $n$ for each $i \in$ $[1, t] ;$ also, let $\Omega$ be a subset of $\Gamma$, and let $\alpha_{1}, \alpha_{2}, \ldots \alpha_{t}$ be non-negative integers such that $\sum_{i=1}^{t} \alpha_{i}=|\Omega|$. If there exists an $|\Omega| \times \ell$ matrix $A$ with $\ell \geq 3$ and entries in $\Omega$ satisfying the following properties:

(1) for each $i \in[1, t]$ there are $\alpha_{i}$ rows of $A$ whose right-to-left sum is an element of order $n_{i}$ in $\Gamma$,

(2) each column of $A$ is a permutation of $\Omega$,

then $\mathrm{OP}_{t}\left(C_{\ell}[\Omega] ;\left(\ell n_{i}\right) ;\left(\alpha_{i}\right)\right)$ has a solution. Moreover, if we also have that

(3) $\Omega$ is closed under taking negatives,

then $\mathrm{OP}_{t}\left(C_{g}[\Omega] ;\left(g n_{i}\right) ;\left(\alpha_{i}\right)\right)$ has a solution for any $g \geq \ell$ with $g \equiv \ell(\bmod 2)$.

Proof. Let $A=\left[a_{h k}\right]$ be an $|\Omega| \times \ell$ matrix with entries from $\Omega \subseteq \Gamma$ and satisfying conditions (1) and (2); also, set $\sigma_{0}=0, \sigma_{i}=\sum_{j=1}^{i} \alpha_{j}$ and let $\mathcal{R}_{i}=\left[\sigma_{i-1}+1, \sigma_{i}\right]$ for $i \in[1, t]$. Note that the $\mathcal{R}_{i}$ s partition the interval $[1,|\Omega|]$ since by assumption $\sigma_{t}=\sum_{j=1}^{t} \alpha_{j}=|\Omega|$. By condition (1) and reordering rows if necessary, we can index the rows of $A$ whose rightto-left sum is an element of order $n_{i}$ by the elements of $\mathcal{R}_{i}$. Thus, we may assume that the right-to-left sum of the $h$-th row of $A$ is an element of order $n_{i}$ if and only if $h \in \mathcal{R}_{i}$.

For $1 \leq h \leq|\Omega|$ and $1 \leq k \leq \ell$, set $s_{h, 0}=0$ and $s_{h, k}=a_{h, k}+a_{h, k-1}+\cdots+a_{h, 1}$. Note that $s_{h, \ell}$ is the right-to-left sum of the $h$-th row of $A$ and, by the above, $s_{h, \ell}$ has order $n_{i}$ if and only if $h \in \mathcal{R}_{i}$; in this case, $n_{i} s_{h, \ell}=0$ and $\mu s_{h, \ell} \neq 0$ for any $\mu \in\left[1, n_{i}-1\right]$. Therefore, for each $i \in[1, t]$ and $h \in \mathcal{R}_{i}$, the following $\ell n_{i}$-cycle is well defined: 


$$
\begin{aligned}
C^{h}=\left(c_{0}^{h}, c_{1}^{h}, \ldots, c_{n_{i} \ell-1}^{h}\right), \text { where } c_{u+\mu \ell}^{h}=\left(u, s_{h, u}+\mu s_{h, \ell}\right), \text { for } & \\
u & \in[0, \ell-1], \mu \in\left[0, n_{i}-1\right] .
\end{aligned}
$$

We start by showing that $\operatorname{Orb}_{\bar{\Gamma}}\left(C^{h}\right)$, where $\bar{\Gamma}=\{0\} \times \Gamma$, is a $C_{n_{i} \ell}$-factor of $C_{\ell}[n]$. First, note that $C^{h}+\left(0, s_{h, \ell}\right)=C^{h}$; in fact, $c_{w}^{h}+\left(0, s_{h, \ell}\right)=c_{w+\ell}^{h}$, for each $w \in\left[0, n_{i} \ell-1\right]$, where the subscript $w+\ell$ is taken modulo $n_{i} \ell$. In other words, addition by $\left(0, s_{h, \ell}\right)$ is equivalent to a rotation of $C^{h}$ by $\ell$. This means that $\left(0, s_{h, \ell}\right)$ lies in $\operatorname{Stab}_{\bar{\Gamma}}\left(C^{h}\right)$. Since the order of $\left(0, s_{h, \ell}\right)$ coincides with the order of $s_{h, \ell}$, which by assumption is $n_{i}$, we have that $\left|\operatorname{Stab}_{\bar{\Gamma}}\left(C^{h}\right)\right| \geq n_{i}$. Therefore,

$$
\left|\operatorname{Orb}_{\bar{\Gamma}}\left(C^{h}\right)\right|=|\bar{\Gamma}| /\left|\operatorname{Stab}_{\bar{\Gamma}}\left(C^{h}\right)\right| \leq n / n_{i} .
$$

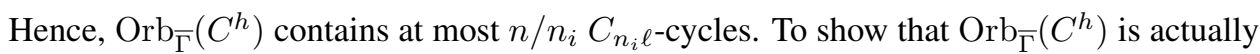
a $C_{n_{i} \ell}$-factor of $C_{\ell}[n]$, it is then enough to check that it contains all vertices of $C_{\ell}[n]$ at least once. Given the point $(u, z) \in \mathbb{Z}_{\ell} \times \Gamma$, we have that $z=s_{h, u}+x_{u}$, for a suitable $x_{u} \in \Gamma$. Therefore, $(u, z)=c_{u}^{h}+\left(0, x_{u}\right)$; hence, $(u, z)$ is a vertex of $C^{h}+\left(0, x_{u}\right) \in \operatorname{Orb}_{\bar{\Gamma}}\left(C^{h}\right)$.

We claim that $\mathcal{F}=\left\{\operatorname{Orb}_{\bar{\Gamma}}\left(C^{h}\right)|h=1,2, \ldots,| \Omega \mid\right\}$ is a 2-factorization of $C_{\ell}[\Omega]$. Note that the factors of $\mathcal{F}$ contain between them at most $\ell n|\Omega|=\left|E\left(C_{\ell}[\Omega]\right)\right|$ edges, counted with their multiplicity. Therefore, it is enough to show that every edge of $C_{\ell}[\Omega]$ lies in some translate of $C^{h}$, for a suitable $h$. First recall that each edge of $C_{\ell}[\Omega]$ has the form $(u, x)(1+u, \omega+x)$ for some $(u, x) \in \mathbb{Z}_{\ell} \times \Gamma$ and $\omega \in \Omega$. Since, by assumption, any column of $A=\left[a_{h k}\right]$ is a permutation of $\Omega$, there is an integer $h$ such that $a_{h, u+1}=\omega$. Note that $\left(u, s_{h, u}\right)\left(1+u, s_{h, u+1}\right) \in E\left(C^{h}\right)$ and $s_{h, u+1}-s_{h, u}=a_{h, u+1}=\omega$. Therefore, $(u, x)(1+u, \omega+x)$ is an edge of $C^{h}+\left(0,-s_{h, u}+x\right)$ and the assertion follows.

In order to prove the second part, let $g=\ell+2 q, \Omega=\left\{\omega_{1}, \omega_{2}, \ldots, \omega_{|\Omega|}\right\}$, and let $A^{\prime}$ be the $|\Omega| \times 2 q$ matrix defined below:

$$
A^{\prime}=\left[\begin{array}{ccccc}
\omega_{1} & -\omega_{1} & \ldots & \omega_{1} & -\omega_{1} \\
\omega_{2} & -\omega_{2} & \ldots & \omega_{2} & -\omega_{2} \\
\vdots & \vdots & & \vdots & \vdots \\
\omega_{|\Omega|} & -\omega_{|\Omega|} & \ldots & \omega_{|\Omega|} & -\omega_{|\Omega|}
\end{array}\right] .
$$

Since $\Omega=-\Omega$ (condition (3)), it is easy to check that the matrix $\left[\begin{array}{ll}A & A^{\prime}\end{array}\right]$ is an $|\Omega| \times g$ matrix satisfying conditions $(1)-(2)$, and this completes the proof.

We point out that while the above theorem is proved for an arbitrary group $\Gamma$, in this paper it is always used when $\Gamma \cong \mathbb{Z}_{n}$. Also, note that if $t=1$, then Theorem 2.1 constructs a $C_{\ell n_{1}}$-factorization of $C_{\ell}[T]$ or a $C_{g n_{1}}$-factorization of $C_{g}[T]$.

The following corollary is a straightforward consequence of the above theorem by taking $\Omega=\Gamma=\mathbb{Z}_{n}$.

Corollary 2.2. Let $t \geq 1$ and let $1 \leq n_{1}<n_{2}<\cdots<n_{t} \leq n$ be odd integers such that $n_{i}$ is a divisor of $n$ for any $i \in[1, t]$; also, let $\alpha_{1}, \alpha_{2}, \ldots, \alpha_{t}$ be non-negative integers such that $\sum_{i=1}^{t} \alpha_{i}=n$. If there exists an $n \times \ell$ matrix $A$ with $\ell \geq 3$ and entries from $\mathbb{Z}_{n}$ satisfying the following properties:

(1) for each $i \in[1, t]$, A has $\alpha_{i}$ rows each of which sums to an element of order $n_{i}$ in $\mathbb{Z}_{n}$,

(2) each column of $A$ is a permutation of $\mathbb{Z}_{n}$, 
then $\mathrm{OP}_{t}\left(C_{g}[n] ;\left(g n_{i}\right) ;\left(\alpha_{i}\right)\right)$ has a solution for any $g \geq \ell$ with $g \equiv \ell(\bmod 2)$.

We end this section by recalling the following result proven in [21] which is here stated in a slightly different, but equivalent, form.

Lemma 2.3 ([21]). Let $\Gamma=\left\{\gamma_{1}, \gamma_{2}, \ldots, \gamma_{n}\right\}$ be an additive abelian group of order $n$, and let $\delta_{1}, \delta_{2}, \ldots, \delta_{n}$ be elements of $\Gamma$, not necessarily distinct, such that $\sum_{i=1}^{n} \delta_{i}=0$. Then there exist a permutation $\Psi$ of $\Gamma$ and a permutation $\pi$ of the interval $[1, n]$ such that $\Psi\left(\gamma_{i}\right)-\gamma_{i}=\delta_{\pi(i)}$ for every $i \in[1, n]$.

\section{Solving $\mathrm{OP}_{t}\left(C_{g}[n] ;\left(g n_{i}\right) ;\left(\alpha_{i}\right)\right)$}

In this section, by exploiting our preliminary results, we provide sufficient conditions for $\mathrm{OP}_{t}\left(C_{g}[n] ;\left(g n_{i}\right) ;\left(\alpha_{i}\right)\right)$ to be solvable.

Theorem 1.4. Let $t \geq 1$ and let $1 \leq n_{1}<n_{2}<\cdots<n_{t} \leq n$ be odd integers such that $n_{i}$ is a divisor of $n$ for each $i \in[1, t]$. Then $\mathrm{OP}_{t}\left(C_{g}[n] ;\left(g n_{i}\right) ;\left(\alpha_{i}\right)\right)$ has a solution whenever $g \geq 3, \sum_{i=1}^{t} \alpha_{i}=n$, and $\alpha_{i} \geq 2$ for every $i \in[1, t]$.

Proof. Let $\alpha_{i} \geq 2$ for $i \in[1, t]$ be integers such that $\sum_{i=1}^{t} \alpha_{i}=n$. Also, let $\Delta=$ $\left\{\delta_{1}, \delta_{2}, \ldots, \delta_{n}\right\}$ be the list of elements of $\mathbb{Z}_{n}$ defined as follows: set $s_{0}=0, s_{i}=\sum_{j=1}^{i} \alpha_{j}$ for every $i \in[1, t]$, and let

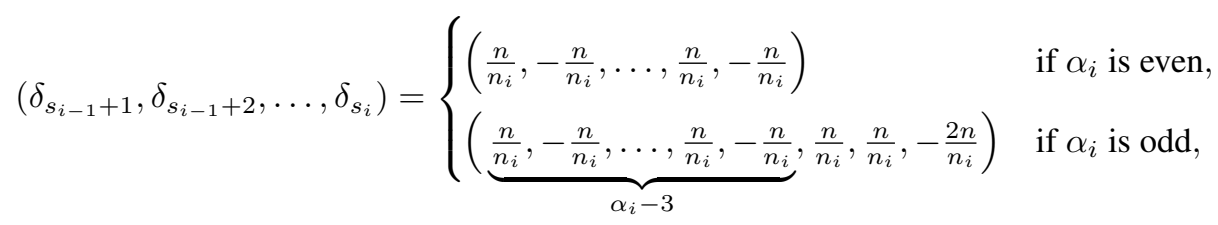

for every $i \in[1, t]$. By recalling that $n$ is odd, we have that $\delta_{s_{i-1}+1}, \delta_{s_{i-1}+2}, \ldots, \delta_{s_{i}}$ are all elements of $\mathbb{Z}_{n}$ of order $n_{i}$, and they sum to 0 . It follows that the elements of $\Delta$ sum to 0 , and Lemma 2.3 guarantees the existence of two permutations $\Psi$ and $\pi$ of $\mathbb{Z}_{n}$ such that $\Psi(i)-i=\delta_{\pi(i)}$ for every $i \in \mathbb{Z}_{n}$.

Now for each $\ell \in\{3,4\}$, let $A_{\ell}$ be the $n \times \ell$ matrix whose $i$-th row is either

$$
\left[\begin{array}{lll}
\Psi(i) & -\frac{i}{2} & -\frac{i}{2}
\end{array}\right] \text { or }\left[\begin{array}{llll}
\Psi(i) & i & -i & -i
\end{array}\right]
$$

according to whether $\ell=3$, or 4 , respectively. It is not difficult to check that $A_{3}$ and $A_{4}$ satisfy the following conditions:

(i) for each $i \in[1, t], A_{3}$ (resp., $A_{4}$ ) has $\alpha_{i}$ rows each of which sums to an element of order $n_{i}$,

(ii) each column of $A_{3}$ (resp., $A_{4}$ ) is a permutation of $\mathbb{Z}_{n}$.

In other words, $A_{3}$ and $A_{4}$ satisfy the assumptions of Corollary 2.2 which guarantees the solvability of $\mathrm{OP}_{t}\left(C_{g}[n] ;\left(g n_{i}\right) ;\left(\alpha_{i}\right)\right)$ whenever $g \geq 3$.

We point out that Theorem 1.4 holds also when $g=2$. In this case, $C_{2}[n]$ is taken to be the complete bipartite graph with parts of size $n$ whose edges are taken with multiplicity two. This can be seen by following the proof of Theorem 1.4 but using the matrix $\left[\begin{array}{ll}\Psi(i) & -i\end{array}\right]$. 


\section{Solving $\mathrm{OP}_{t}\left(v ;\left(N_{i}\right) ;\left(\alpha_{i}\right)\right)$}

We say that $\mathrm{OP}_{t}\left(G ;\left(N_{i}\right) ;\left(\alpha_{i}\right)\right)$ and $\mathrm{OP}_{u}\left(G ;\left(M_{j}\right) ;\left(\beta_{j}\right)\right)$ are equivalent if $\sum_{N_{i}=x} \alpha_{i}=$ $\sum_{M_{j}=x} \beta_{j}$ for any $x \geq 3$. For example, $\mathrm{OP}_{4}(G ; 4,4,5,7 ; 4,6,8,2)$ is equivalent to $\mathrm{OP}_{5}(G ; 4,4,4,5,7 ; 2,3,5,8,2)$.

Moreover, for any non-negative integer $\alpha$ we define the integer $f(\alpha)$ as follows:

$$
f(\alpha)=\frac{\alpha-\rho}{3}, \quad \text { where }\{0,2,4\} \ni \rho \equiv \alpha \quad(\bmod 3) .
$$

Clearly, $\alpha=3 f(\alpha)+\rho$ and $f(\alpha) \equiv \alpha(\bmod 2)$.

The following result provides sufficient conditions for the existence of a solution to $\mathrm{OP}_{t}\left(G ;\left(g n_{i}\right) ;\left(\alpha_{i}\right)\right)$ for an arbitrary graph $G$.

Theorem 4.1. Let $t \geq 2$, and let $1 \leq n_{1}<n_{2}<\cdots<n_{t} \leq n$ be odd integers such that $n_{i}$ is a divisor of $n$ for each $i \in[1, t]$. Also, let $G$ be a graph having a factorization into $r C_{g}[n]$-factors with $g \geq 3$. Then, $\mathrm{OP}_{t}\left(G ;\left(g n_{i}\right) ;\left(\alpha_{i}\right)\right)$ has a solution whenever the following conditions simultaneously hold:

(1) $\sum_{i=1}^{t} \alpha_{i}=r n$;

(2) $0 \leq \alpha_{i} \neq 1$ for every $i \in[1, t]$;

(3) $\sum_{i=1}^{t} f\left(\alpha_{i}\right) \geq r$;

(4) $\mid\left\{i \in[1, t] \mid \alpha_{i}\right.$ is odd $\} \mid \leq r\left(2\left\lfloor\frac{n-2}{6}\right\rfloor+1\right)$.

Proof. Let $n=6 q+\rho$ where $\rho \in\{3,5,7\}$ and let $\mathcal{F}=\left\{F_{1}, F_{2}, \ldots, F_{r}\right\}$ be a factorization of $G$ into $r C_{g}[n]$-factors. We proceed by induction on $r$. If $r=1$, the assertion follows from Theorem 1.4. Now, let $r \geq 2$ and assume that the assertion holds for any graph having a factorization into $r-1 C_{g}[n]$-factors. It is enough to show that $\mathrm{OP}_{t}\left(G ;\left(g n_{i}\right) ;\left(\alpha_{i}\right)\right)$ is equivalent to a problem of the following form:

$$
\begin{aligned}
\mathrm{OP}_{u}\left(G ;\left(N_{j}\right) ;\left(\beta_{j}\right)\right), \quad \text { where } \beta_{j} & \in\{2,3\} \text { and } \\
r & \leq \delta=\left|\left\{j \in[1, u] \mid \beta_{j}=3\right\}\right| \leq r(2 q+1) .
\end{aligned}
$$

In fact, assuming this equivalence, we only need define $\overline{\beta_{j}}$ s so that $\mathrm{OP}_{u}\left(F_{1} ;\left(N_{j}\right) ;\left(\overline{\beta_{j}}\right)\right)$ and $\mathrm{OP}_{u}\left(G-F_{1} ;\left(N_{j}\right) ;\left(\beta_{j}-\overline{\beta_{j}}\right)\right)$ are solvable; it follows that the problem in (4.1), and hence, the original problem has a solution. We first assume (without loss of generality) that $\beta_{j}=3$ if and only if $j \in[1, \delta]$ and consider the following two cases:

1. if $\delta \in[r, r+2 q]$, set

$$
\overline{\beta_{j}}= \begin{cases}\beta_{j} & \text { if } j \in\{1\} \cup\left[\delta+1, \delta+\frac{n-3}{2}\right] \\ 0 & \text { otherwise }\end{cases}
$$

2. if $\delta \in[r+2 q+1, r(2 q+1)]$, we define $\overline{\beta_{j}}$ as follows,

$$
\overline{\beta_{j}}= \begin{cases}\beta_{j} & \text { if } j=[1,2 q+1] \cup\left[\delta+1, \delta+\frac{\rho-3}{2}\right] \\ 0 & \text { otherwise. }\end{cases}
$$


By Theorem 1.4, there exists a solution to $\mathrm{OP}_{u}\left(F_{1} ;\left(N_{j}\right) ;\left(\overline{\beta_{j}}\right)\right)$. It is not difficult to check that $\mathrm{OP}_{u}\left(G-F_{1} ;\left(N_{j}\right) ;\left(\beta_{j}-\overline{\beta_{j}}\right)\right)$ satisfies all the assumption of this theorem, therefore, by the induction hypothesis, it is solvable.

We now show that $\mathrm{OP}_{t}\left(G ;\left(g n_{i}\right) ;\left(\alpha_{i}\right)\right)$ is equivalent to a problem of the form (4.1). We reorder the $\alpha_{i}$ s so that the even $\alpha_{i}$ s appear first. For every $i \in[1, t]$ we define the quadruple of integers $\left(\gamma_{2 i-1}, \gamma_{2 i}, N_{2 i-1}, N_{2 i}\right)$ as follows:

$$
\left(\gamma_{2 i-1}, \gamma_{2 i}\right)=\left\{\begin{array}{ll}
\left(\alpha_{i}-3,3\right) & \text { if } \alpha_{i} \text { is odd; } \\
\left(\alpha_{i}, 0\right) & \text { if } \alpha_{i} \text { is even; }
\end{array} \quad N_{2 i-1}=N_{2 i}=g n_{i} .\right.
$$

It follows that $\gamma_{1}, \gamma_{2}, \ldots, \gamma_{2 t-d}$ are even, whereas $\gamma_{i}=3$ for any $i \in[2 t-d+1,2 t]$, where $d=\mid\left\{i \in[1, t] \mid \alpha_{i}\right.$ is odd $\} \mid$ is the number of odd $\alpha_{i}$ s. We point out that $\mathrm{OP}_{t}\left(G ;\left(g n_{i}\right) ;\left(\alpha_{i}\right)\right)$ is equivalent to $\mathrm{OP}_{2 t}\left(G ;\left(N_{i}\right) ;\left(\gamma_{i}\right)\right)$; also, since by assumption $\sum_{i=1}^{t} f\left(\alpha_{i}\right) \geq r$, it follows that $\sum_{i=1}^{2 t} f\left(\gamma_{i}\right) \geq r$.

We first assume that $d<r$. Now, let $k \in[1,2 t-d]$ be the greatest integer such that $\sum_{i=k}^{2 t} f\left(\gamma_{i}\right) \geq r$, and set $r^{\prime}=\sum_{i=k+1}^{2 t} f\left(\gamma_{i}\right)$. Clearly, $r^{\prime}<r$; also, $r-r^{\prime}$ is even, since:

$$
r \equiv r n=\sum_{i=1}^{k} \gamma_{i}+\sum_{i=k+1}^{2 t} \gamma_{i} \equiv \sum_{i=k+1}^{2 t} \gamma_{i} \equiv \sum_{i=k+1}^{2 t} f\left(\gamma_{i}\right)=r^{\prime} \quad(\bmod 2)
$$

We proceed by defining a suitable partition $\left(\gamma_{i 1}, \gamma_{i 2}, \ldots, \gamma_{i, t_{i}}\right)$ of the integer $\gamma_{i}$ such that $\gamma_{i j} \in\{0,2,3\}$. First, for each $i \in[k, 2 t]$ set $\left(q_{i}, \rho_{i}\right)=\left(f\left(\gamma_{i}\right), \gamma_{i}-3 f\left(\gamma_{i}\right)\right)$ and note that $\rho_{i} \in\{0,2,4\}$. Recall now that $\gamma_{k}=3 q_{k}+\rho_{k}$ is even, hence $q_{k}$ is even; also, $r-r^{\prime}$ is even and $q_{k} \geq r-r^{\prime}$. Therefore, $\gamma_{k}=3\left(r-r^{\prime}\right)+2 y$ where $y=\frac{3\left(q_{k}-r+r^{\prime}\right)+\rho_{k}}{2}$. We now define a partition $\left(\gamma_{i 1}, \gamma_{i 2}, \ldots, \gamma_{i, t_{i}}\right)$ of $\gamma_{i}$ as follows:

- if $i \in[1, k-1]$, set $t_{i}=\gamma_{i} / 2$ and $\gamma_{i j}=2$ for any $j \in\left[1, t_{i}\right]$;

- if $i=k$, set $t_{i}=r-r^{\prime}+y$ and $\gamma_{i j}= \begin{cases}3 & \text { if } j \in\left[1, r-r^{\prime}\right] \text {; } \\ 2 & \text { otherwise. }\end{cases}$

- if $i \in[k+1,2 t]$, set $t_{i}=q_{i}+2$ and

$$
\gamma_{i j}= \begin{cases}3 & \text { if } j \in\left[1, q_{i}\right] \\ 0 & \text { if }\left(j, \rho_{i}\right) \in\left\{\left(q_{i}+1,0\right),\left(q_{i}+2,0\right),\left(q_{i}+2,2\right)\right\} \\ 2 & \text { otherwise }\end{cases}
$$

Finally, for any $i \in[1,2 t]$ and $j \in\left[1, t_{i}\right]$ set $N_{i j}=N_{i}$ and $u=\sum_{i=1}^{2 t} t_{i}$. Clearly, the original problem $\mathrm{OP}_{2 t}\left(G ;\left(N_{i}\right) ;\left(\gamma_{i}\right)\right)$ is equivalent to $\mathrm{OP}_{u}\left(G ;\left(N_{i j}\right) ;\left(\gamma_{i j}\right)\right)$ where $\gamma_{i j} \in$ $\{0,2,3\}$ and there are exactly $r \gamma_{i j}$ s equal to 3 . By removing all pairs $\left(N_{i j}, \gamma_{i j}\right)$ with $\gamma_{i j}=0$, we obtain a problem of the form (4.1).

We finally consider the case where $d \geq r$. As before, we define a partition $\left(\gamma_{i 1}, \gamma_{i 2}, \ldots, \gamma_{i, t_{i}}\right)$ of the integer $\gamma_{i}$ as follows:

$$
\left(t_{i}, \gamma_{i j}\right)= \begin{cases}\left(\frac{\gamma_{i}}{2}, 2\right) & \text { if } i \in[1,2 t-d] \text { and } j \in\left[1, \frac{\gamma_{i}}{2}\right] \\ (1,3) & \text { otherwise }\end{cases}
$$


and set $N_{i j}=N_{i}$ for any $j \in\left[1, t_{i}\right]$, and $u=\sum_{i=1}^{2 t} t_{i}$. Clearly, the original problem $\mathrm{OP}_{2 t}\left(G ;\left(N_{i}\right) ;\left(\gamma_{i}\right)\right)$ is equivalent to $\mathrm{OP}_{u}\left(G ;\left(N_{i j}\right) ;\left(\gamma_{i j}\right)\right)$ where $\gamma_{i j} \in\{2,3\}$ and there are exactly $d \gamma_{i j}$ s equal to 3 . Since, $d \leq r(2 q+1)$ by assumption, then $\mathrm{OP}_{u}\left(G ;\left(N_{i j}\right) ;\left(\gamma_{i j}\right)\right)$ is of the form (4.1), and this completes the proof.

We now provide a result for the complete equipartite graph.

Theorem 4.2. Let $s, w \geq 3$ be odd integers, let $3 \leq N_{1}<N_{2}<\cdots<N_{t}$, and let $\alpha_{1}, \alpha_{2}, \ldots, \alpha_{t}$ be positive integers. If $\sum_{i=1}^{t} \alpha_{i}=\frac{(s-1) w}{2}$ and each $N_{i}$ is a divisor of $w$, then $\mathrm{OP}_{t}\left(K_{s}[w] ;\left(N_{i}\right) ;\left(\alpha_{i}\right)\right)$ is solvable, except possibly when $t>1$ and at least one of the following conditions is satisfied:

(A) $\alpha_{i}=1$ for some $i \in[1, t]$;

(B) $\operatorname{gcd}\left(N_{1}, N_{2}, \ldots, N_{t}\right)=1$.

Proof. We assume that $t \geq 2$, since the case $t=1$ is solved in Theorem 1.1.

Now, set $N=\operatorname{lcm}\left(N_{1}, N_{2}, \ldots, N_{t}\right)$ and $g=\operatorname{gcd}\left(N_{1}, N_{2}, \ldots, N_{t}\right)$; also, let $n_{i}=$ $N_{i} / g$, set $n=\operatorname{lcm}\left(n_{1}, n_{2}, \ldots, n_{t}\right)$ and note that $N=g n$. By assumption, we have that each $N_{i}$ is a divisor of $w$, that is, $N$ is a divisor of $w$, hence $w=g n \bar{w}$ for some integer $\bar{w}>0$. By Theorem 1.1, there exists a $C_{g}$-factorization of $K_{s}[g \bar{w}]$ with $r C_{g}$-factors, where $r=g \bar{w}(s-1) / 2$. By expanding each vertex of this factorization by $n$, we get a $C_{g}[n]$-factorization $\mathcal{F}$ of $K_{s}[g \bar{w}][n] \cong K_{s}[w]$ with $r C_{g}[n]$-factors.

We first assume that $n \geq 7$. In this case, to solve $\mathrm{OP}_{t}\left(K_{s}[w] ;\left(N_{i}\right) ;\left(\alpha_{i}\right)\right)$ it is enough to show that conditions (1) $-(4)$ of Theorem 4.1 are satisfied. By assumption $\sum_{i=1}^{t} \alpha_{i}=$ $\frac{(s-1) w}{2}=r n$, and by exception (A) we have that $\alpha_{i} \geq 2$ for every $i \in[1, t]$. Further,

$$
r\left(2\left\lfloor\frac{n-2}{6}\right\rfloor+1\right) \geq \frac{r(n-4)}{3}=\frac{g \bar{w}(s-1)}{6}(n-4) \geq n-4 \geq \frac{n}{3},
$$

and since $n$ has at most $\left\lfloor\frac{n}{3}\right\rfloor$ distinct divisors, we have that $\frac{n}{3} \geq t$, hence $r\left(2\left\lfloor\frac{n-2}{6}\right\rfloor+1\right) \geq$ $t$. Finally, we have that

$$
r n=\sum_{i=1}^{t} \alpha_{i} \leq \sum_{i=1}^{t}\left(3 f\left(\alpha_{i}\right)+4\right)=4 t+3 \sum_{i=1}^{t} f\left(\alpha_{i}\right)<4 r+3 \sum_{i=1}^{t} f\left(\alpha_{i}\right),
$$

and since $n \geq 7$, it follows that $\sum_{i=1}^{t} f\left(\alpha_{i}\right)>r(n-4) / 3 \geq r$. Therefore, all conditions of Theorem 4.1 are satisfied, hence $\mathrm{OP}_{t}\left(K_{s}[w] ;\left(N_{i}\right) ;\left(\alpha_{i}\right)\right)$ is solvable.

It is left to consider the cases where $n \in\{3,5\}$. Since $N_{i}$ is a multiple of $g$ and a divisor of $g n$, then $N_{i} \in\{g, g n\}$ for any $i$. By recalling that $N_{1}<N_{2}<\cdots<N_{t}$ and $t \geq 2$, we have that $t=2$ and $\left(N_{1}, N_{2}\right)=(g, g n)$. Now, let $\alpha_{2}=x n+y$ where $x \geq 0$ and $y \in[0, n-1]$, and since $\alpha_{2} \geq 2$ (exception (A)), then $(x, y) \neq(0,1)$. If $y \neq 1$, we apply Theorem 1.4 to fill $x C_{g}[n]$-factors of $\mathcal{F}$ with a solution of $\mathrm{OP}_{2}\left(C_{g}[n] ; g, g n ; 0, n\right)$, one $C_{g}[n]$-factor with a solution of $\mathrm{OP}_{2}\left(C_{g}[n] ; g, g n ; n-y, y\right)$, and the remaining $r-x-1$ factors of $\mathcal{F}$ with a solution of $\mathrm{OP}_{2}\left(C_{g}[n] ; g, g n ; n, 0\right)$. Similarly, if $y=1$, since $x>0$ and $r \geq g \geq 3$ (exception (B)), we again apply Theorem 1.4 and fill $x-1 C_{g}[n]$ factors of $\mathcal{F}$ with a solution of $\mathrm{OP}_{2}\left(C_{g}[n] ; g, g n ; 0, n\right)$, one $C_{g}[n]$-factor with a solution of $\mathrm{OP}_{2}\left(C_{g}[n] ; g, g n ; 1, n-1\right)$, one $C_{g}[n]$-factor with a solution of $\mathrm{OP}_{2}\left(C_{g}[n] ; g, g n\right.$; $n-2,2)$, and the remaining $r-x-1$ factors of $\mathcal{F}$ with a solution of $\mathrm{OP}_{2}\left(C_{g}[n] ; g, g n ; n, 0\right)$. 
We are now ready to prove the main result of this paper.

Theorem 1.3. Let $v \geq 3$ be odd, let $3 \leq N_{1}<N_{2}<\cdots<N_{t}$ and set $N=$ $\operatorname{lcm}\left(N_{1}, N_{2}, \ldots, N_{t}\right)$ and $g=\operatorname{gcd}\left(N_{1}, N_{2}, \ldots, N_{t}\right) ;$ also, let $\alpha_{1}, \alpha_{2}, \ldots, \alpha_{t}$ be positive integers. Then, $\mathrm{OP}_{t}\left(v ;\left(N_{i}\right) ;\left(\alpha_{i}\right)\right)$ has a solution if and only if $N$ is a divisor of $v$ and $\sum_{i=1}^{t} \alpha_{i}=\frac{v-1}{2}$ except possibly when $t>1$ and at least one of the following conditions is satisfied:

(I) $\alpha_{i}=1$ for some $i \in[1, t]$;

(II) $\alpha_{i} \in\left[2, \frac{N-3}{2}\right] \cup\left\{\frac{N+1}{2}\right\}$ for every $i \in[1, t]$;

(III) $g=1$;

(IV) $v=N$.

Proof. By Theorem 1.2, if $\mathrm{OP}_{t}\left(v ;\left(N_{i}\right) ;\left(\alpha_{i}\right)\right)$ has a solution, then $N$ is a divisor of $v$ and $\sum_{i=1}^{t} \alpha_{i}=\frac{v-1}{2}$. We now show sufficiency and assume that $t \geq 2$, since the case $t=1$ is solved in Theorem 1.1. Let $v=N s$ for a suitable odd integer $s$. By exception (IV), we have that $s \geq 3$.

We first factorize $K_{v}$ into $G_{0}=s K_{N}$ and $G_{1}=K_{s}[N]$. By exception (II), there exists $k \in[1, t]$ such that either $\alpha_{k}=\frac{N-1}{2}$ or $\alpha_{i} \geq \frac{N+3}{2}$. Then, we apply Theorem 1.1 to fill $G_{0}$ with a $C_{N_{k}}$-factorization. It remains to solve $\mathrm{OP}_{t}\left(G_{1} ;\left(N_{i}\right) ;\left(\overline{\alpha_{i}}\right)\right)$ where $\overline{\alpha_{i}}=\alpha_{i}-\frac{N-1}{2}$ if $i=k$, and $\overline{\alpha_{i}}=\alpha_{i}$ otherwise. By taking into account exceptions (I) and (III), we have that:

(a) $\overline{\alpha_{i}} \neq 1$ for any $i \in[1, t]$, and

(b) $g \geq 3$.

Therefore, Theorem 4.2 guarantees the solvability of $\mathrm{OP}_{t}\left(G_{1} ;\left(N_{i}\right) ;\left(\overline{\alpha_{i}}\right)\right)$ and the assertion is proven.

Corollary 4.3. Let $v \geq 3$ be odd, let $3 \leq N_{1}<N_{2}<\cdots<N_{t}$, set $N=\operatorname{lcm}\left(N_{1}\right.$, $\left.N_{2}, \ldots, N_{t}\right)$, and let $\alpha_{1}, \alpha_{2}, \ldots, \alpha_{t}$ be positive integers. Then, $\mathrm{OP}_{t}\left(v ;\left(N_{i}\right) ;\left(\alpha_{i}\right)\right)$ has $a$ solution whenever $N$ is a divisor of $v, \sum_{i=1}^{t} \alpha_{i}=\frac{v-1}{2}$, and the following conditions are satisfied:

(1) $\alpha_{i} \neq 1$ for any $i \in[1, t]$;

(2) $\operatorname{gcd}\left(N_{1}, N_{2}, \ldots, N_{t}\right) \geq 3$;

(3) $v \geq(t+1) N$.

Proof. The case $t=1$ is solved in Theorem 1.1, therefore, we let $t \geq 2$. By condition (3) and considering that $\sum_{i=1}^{t} \alpha_{i}=\frac{v-1}{2}$, it follows that there exists $\bar{k} \in[1, t]$ such that $\alpha_{k} \geq \frac{N+3}{2}$. If we also take into account conditions (1) and (2), we have that all assumptions of Theorem 1.3 are satisfied, and the assertion follows.

\section{Conclusions}

This paper deals with the generalized Oberwolfach problem, denoted by $\mathrm{OP}_{t}\left(v ; N_{1}, N_{2}\right.$, $\left.\ldots, N_{t} ; \alpha_{1}, \alpha_{2}, \ldots, \alpha_{t}\right)$, which asks for a 2-factorization of the complete graph $K_{v}$ into $\alpha_{i}$ copies of a $C_{N_{i}}$-factor, for $i \in\{1,2, \ldots, t\}$. For a solution of this problem to exist, $v$ must be odd, each $N_{i}$ must be a divisor of $v$, and $\sum_{i} \alpha_{i}=\frac{v-1}{2}$ (Theorem 1.2). 
This problem has been widely studied when $t=1$ or 2 . The case $t=1$ represents the 'uniform' Oberwolfach problem which has been solved in 1989 [3]. When $t=2$, this problem is known as the Hamilton-Waterloo problem. Although this version of the problem is still open, by using techniques similar to those adopted in this paper, the current authors were able to make significant progress in the challenging case where the cycle lengths are odd $[12,13]$.

This paper makes significant progress (Theorem 1.3) on the generalized Oberwolfach problem by showing that the above necessary conditions suffice whenever $v>(t+1) N$, each $\alpha_{i}$ is greater than 1 , and $g \geq 3$, where $g=\operatorname{gcd}\left(N_{1}, N_{2}, \ldots, N_{t}\right)$ (Corollary 4.3). This result and its stronger version (Theorem 1.3) rely on Theorem 1.4 which concerns the existence of a factorization of $C_{g}[n]$ into $\alpha_{i} C_{g n_{i}}$-factors for $i \in\{1,2, \ldots, t\}$ (that is, the generalized Oberwolfach problem over $C_{g}[n]$ ). Theorem 1.4 shows that the trivial necessary conditions suffice whenever $g \geq 3$, and $\alpha_{i}>1$ for each $i$. Clearly, removing this last condition from Theorem 1.4 would automatically yield a similar improvement of our main theorem.

More generally, we provide sufficient conditions (Theorem 4.1) for the solvability of the generalized Oberwolfach problem over an arbitrary graph $G$. As a consequence, we provide, with Theorem 4.2, a result for the complete equipartite graph, similar to those mentioned above.

\section{References}

[1] P. Adams and D. Bryant, Two-factorisations of complete graphs of orders fifteen and seventeen, Australas. J. Combin. 35 (2006), 113-118, https: / / j jc.maths . uq. edu.au/ pdf/35/ajc_v35_p113.pdf.

[2] B. Alspach and R. Häggkvist, Some observations on the Oberwolfach problem, J. Graph Theory 9 (1985), 177-187, doi:10.1002/jgt.3190090114.

[3] B. Alspach, P. J. Schellenberg, D. R. Stinson and D. Wagner, The Oberwolfach problem and factors of uniform odd length cycles, J. Comb. Theory Ser. A 52 (1989), 20-43, doi:10.1016/ 0097-3165(89)90059-9.

[4] J. Asplund, D. Kamin, M. Keranen, A. Pastine and S. Özkan, On the Hamilton-Waterloo problem with triangle factors and $C_{3 x}$-factors, Australas. J. Combin. 64 (2016), 458-474, https://ajc.maths.uq.edu.au/pdf/64/ajc_v64_p458.pdf.

[5] D. Bryant, Hamilton cycle rich two-factorizations of complete graphs, J. Combin. Des. 12 (2004), 147-155, doi:10.1002/jcd.20005.

[6] D. Bryant and P. Danziger, On bipartite 2-factorizations of $K_{n}-I$ and the Oberwolfach problem, J. Graph Theory 68 (2011), 22-37, doi:10.1002/jgt.20538.

[7] D. Bryant, P. Danziger and M. Dean, On the Hamilton-Waterloo problem for bipartite 2-factors, J. Combin. Des. 21 (2013), 60-80, doi:10.1002/jcd.21312.

[8] D. Bryant, P. Danziger and W. Pettersson, Bipartite 2-factorizations of complete multipartite graphs, J. Graph Theory 78 (2015), 287-294, doi:10.1002/jgt.21806.

[9] D. Bryant and V. Scharaschkin, Complete solutions to the Oberwolfach problem for an infinite set of orders, J. Comb. Theory Ser. B 99 (2009), 904-918, doi:10.1016/j.jctb.2009.03.003.

[10] M. Buratti and P. Danziger, A cyclic solution for an infinite class of Hamilton-Waterloo problems, Graphs Combin. 32 (2016), 521-531, doi:10.1007/s00373-015-1582-x.

[11] M. Buratti and T. Traetta, 2-starters, graceful labelings, and a doubling construction for the Oberwolfach problem, J. Combin. Des. 20 (2012), 483-503, doi:10.1002/jcd.21296. 
[12] A. C. Burgess, P. Danziger and T. Traetta, On the Hamilton-Waterloo problem with odd orders, J. Combin. Des. 25 (2017), 258-287, doi:10.1002/jcd.21552.

[13] A. C. Burgess, P. Danziger and T. Traetta, On the Hamilton-Waterloo problem with odd cycle lengths, J. Combin. Des. 26 (2018), 51-83, doi:10.1002/jcd.21586.

[14] N. J. Cavenagh, S. I. El-Zanati, A. Khodkar and C. Vanden Eynden, On a generalization of the Oberwolfach problem, J. Comb. Theory Ser. A 106 (2004), 255-275, doi:10.1016/j.jcta.2004. 02.003 .

[15] C. J. Colbourn and J. H. Dinitz (eds.), The CRC Handbook of Combinatorial Designs, CRC Press Series on Discrete Mathematics and its Applications, CRC Press, Boca Raton, Florida, 1996, doi:10.1201/9781420049954.

[16] J. H. Dinitz and A. C. H. Ling, The Hamilton-Waterloo problem: the case of triangle-factors and one Hamilton cycle, J. Combin. Des. 17 (2009), 160-176, doi:10.1002/jcd.20196.

[17] S. I. El-Zanati, S. K. Tipnis and C. Vanden Eynden, A generalization of the Oberwolfach problem, J. Graph Theory 41 (2002), 151-161, doi:10.1002/jgt.10058.

[18] F. Franek, J. Holub and A. Rosa, Two-factorizations of small complete graphs. II. The case of 13 vertices, J. Combin. Math. Combin. Comput. 51 (2004), 89-94.

[19] F. Franek and A. Rosa, Two-factorizations of small complete graphs, J. Statist. Plann. Inference 86 (2000), 435-442, doi:10.1016/s0378-3758(99)00123-8.

[20] S. Glock, F. Joos, J. Kim, D. Kühn and D. Osthus, Resolution of the Oberwolfach problem, arXiv:1806.04644 [math.CO].

[21] M. Hall, Jr., A combinatorial problem on abelian groups, Proc. Amer. Math. Soc. 3 (1952), 584-587, doi:10.2307/2032592.

[22] D. G. Hoffman and P. J. Schellenberg, The existence of $C_{k}$-factorizations of $K_{2 n}-F$, Discrete Math. 97 (1991), 243-250, doi:10.1016/0012-365x(91)90440-d.

[23] M. S. Keranen and A. Pastine, A generalization of the Hamilton-Waterloo problem on complete equipartite graphs, J. Combin. Des. 25 (2017), 431-468, doi:10.1002/jcd.21560.

[24] J. Liu, The equipartite Oberwolfach problem with uniform tables, J. Comb. Theory Ser. A 101 (2003), 20-34, doi:10.1016/s0097-3165(02)00011-0.

[25] F. Merola and T. Traetta, Infinitely many cyclic solutions to the Hamilton-Waterloo problem with odd length cycles, Discrete Math. 339 (2016), 2267-2283, doi:10.1016/j.disc.2016.03. 026.

[26] U. Odabaşı and S. Özkan, Uniformly resolvable cycle decompositions with four different factors, Graphs Combin. 33 (2017), 1591-1606, doi:10.1007/s00373-017-1856-6.

[27] H. E. Rose, A Course on Finite Groups, Universitext, Springer-Verlag, London, 2009, doi: 10.1007/978-1-84882-889-6.

[28] B. Stevens, The anti-Oberwolfach solution: pancyclic 2-factorizations of complete graphs, Theoret. Comput. Sci. 297 (2003), 399-424, doi:10.1016/s0304-3975(02)00650-3.

[29] T. Traetta, A complete solution to the two-table Oberwolfach problems, J. Comb. Theory Ser. A 120 (2013), 984-997, doi:10.1016/j.jcta.2013.01.003. 